\title{
Reporting guidelines for journal and conference abstracts
}

\author{
Jérémie F. Cohen*, PhD \\ - Université de Paris, CRESS, Inserm, INRA, F75004 Paris, France \\ - Department of General Pediatrics and Pediatric Infectious Diseases, Necker Hospital for Sick Children, APHP, \\ Université de Paris, Paris, France
}

Daniël A. Korevaar, PhD

Department of Respiratory Medicine, Amsterdam UMC, University of Amsterdam, Amsterdam, The Netherlands d.a.korevaar@amsterdamumc.nl

Isabelle Boutron, PhD

Université de Paris, CRESS, Inserm, INRA, F75004 Paris, France

isabelle.boutron@aphp.fr

Constantine A. Gatsonis, PhD

Department of Biostatistics, Brown University School of Public Health, Providence, Rhode Island, USA

gatsonis@stat.brown.edu

\section{Sally Hopewell, PhD}

Centre for Statistics in Medicine, Nuffield Department of Orthopaedics, Rheumatology and Musculoskeletal Sciences, University of Oxford, UK

sally.hopewell@csm.ox.ac.uk

\section{Matthew DF Mclnnes, PhD}

Department of Radiology, University of Ottawa; Ottawa Hospital Research Institute, Clinical Epidemiology

Program, Ottawa, Canada

mmcinnes@toh.ca

David Moher, PhD

- Centre for Journalology, Clinical Epidemiology Program, Ottawa Hospital Research Institute, Ottawa, Canada

- School of Epidemiology and Public Health, University of Ottawa, Canada

dmoher@ohri.ca

Erik von Elm, PhD

Cochrane Switzerland, Centre for Primary Care and Public Health, University of Lausanne, Lausanne, Switzerland erik.vonelm@unisante.ch

\section{Patrick M. Bossuyt, PhD}

Department of Clinical Epidemiology, Biostatistics and Bioinformatics, Amsterdam UMC, University of Amsterdam, Amsterdam, The Netherlands

p.m.bossuyt@amsterdamumc.nl

\section{* Correspondence to: Jérémie F. Cohen, MD PhD}

Inserm UMR 1153, Center for Epidemiology and Statistics (CRESS), Université de Paris, Paris, France jeremie.cohen@inserm.fr; ORCID ID: 0000-0003-3572-8985

Article type: Commentary

Word count (text only): 2196 References: 36

Tables: $3 \quad$ Figures: $0 \quad$ Boxes: 1

Keywords: Reporting quality; Guidelines; Research transparency; Checklist; Abstracting and Indexing; EQUATOR network. 


\section{INTRODUCTION}

Clinicians, researchers, systematic reviewers, and policy makers need to screen large amounts of information quickly. Journal and conference abstracts play an essential role in this process. They are condensed scientific reports, most often limited to 300 words or less, and in most cases structured with headings pertaining to background and objectives, methods, results, and conclusions. For many readers, the journal abstract may represent the sole source of information about a study report. Despite the emergence of open access publication models, many journals still operate behind a paywall restricting access to the full article content. Also, editors and conference organisers often base their decisions on the abstract alone. Furthermore, less than half of the studies presented as conference abstracts at biomedical meetings are eventually published in full (1).

Abstracts should be prepared in a manner that allows readers to appreciate the essential elements of the study methods and main results in order to identify potential concerns about study internal validity. This can only be achieved if abstracts are sufficiently informative. Unfortunately, several evaluations have identified deficiencies in the reporting of abstracts across different study types, including randomised trials, systematic reviews, and diagnostic accuracy studies (2-4).

To improve the completeness and informativeness of journal and conference abstracts, several groups have developed specific guidance for reporting abstracts for different types of study designs. The longterm goal of such reporting guidance is to increase the completeness and transparency of published scientific evidence, avoiding research waste (5), while increasing trustworthiness. More than 400 reporting guidelines in a wide range of research fields have been published in the past 20 years, but only a minority present detailed guidance for abstracts. Here we provide an overview of existing reporting guidelines for journal and conference abstracts, with the aim of raising awareness of their existence and potential benefits. 


\section{General characteristics of reporting guidelines for abstracts}

Using the methods described in Box 1, we identified five reporting guidelines for abstracts that were derived from existing reporting guidelines for full study reports: CONSORT (Consolidated standards of reporting trials) $(6,7)$, STROBE (Strengthening the reporting of observational studies in epidemiology) $(8,9)$, PRISMA (Preferred reporting items for systematic reviews and meta-analyses) $(10,11)$, STARD (Standards for reporting diagnostic accuracy studies) $(12,13)$, and PRIO-harms (Preferred reporting items for overviews of systematic reviews including harms) $(14,15)$. These were included in our review of reporting guidelines for abstracts.

We also identified nine extensions of CONSORT (CONSORT for: cluster trials; noninferiority and equivalence trials; N-of-1 trials; pilot and feasibility trials; nonpharmacologic trials; trials of social and psychological interventions; stepped wedge cluster trials; within-person trials; and crossover trials) (16-24) and one extension for PRISMA (PRISMA for diagnostic accuracy studies) (25) that also provided guidance for abstracts. We also identified reporting guidelines for full-text articles that included some specific items about the abstract, but did not provide a full checklist for abstracts (see for example RECORD, a STROBE extension for studies based on routinely-collected health data) (26). These were not in the scope of our review.

A protocol describing the development of the checklist was only available for STARD for Abstracts (Table 1). A detailed consensus procedure was reported in all cases, except for STROBE for Abstracts. Pilot-testing was reported only for 2 out of 5 checklists. Evaluations were scarce: we identified studies of adherence for CONSORT, STROBE, PRISMA, and STARD for Abstracts. Impact studies were found for CONSORT and PRISMA for Abstracts only. We did not identify any assessment of user satisfaction.

The five checklists contain a median of 12 items (range 11-17; Table 2 and Table 3). They share a core set of six generic items covering the critical elements of any study: identification of the study design, study objectives, eligibility criteria, numbers of participants (or studies, in case of a systematic review), results for the primary outcome and its precision, and general interpretation of the study findings. 
There are also five generic items that are included in some but not all of the checklists: clearly defined primary outcome, study setting, study registration number and registry, source of funding, and contact details for the corresponding author (Table 2). Each guideline also points to specific items, such as harms in studies of interventions (Table 3) (7).

\section{CONSORT for Abstracts}

CONSORT for Abstracts is an extension of $\operatorname{CONSORT}(6,7)$, a guideline for reporting the results of randomised controlled trials. Specific items include trial design (parallel, cluster, or non-inferiority), a description of the interventions intended for each group, information about the method of random allocation, blinding of participants and outcome assessors, important adverse events or side effects, and whether trial recruitment was active or completed.

So far, nine extensions of CONSORT developed for addressing specific types of trials also provide some elements of guidance for abstracts (16-24); these were not in the scope of our review. In 2017, for example, the CONSORT Group published an updated extension for reporting the results of randomised trials of nonpharmacologic treatments, such as surgery or psychotherapy (20). This extension also presents an extension to CONSORT for Abstracts, with explicit guidance for the content of abstracts of nonpharmacologic trials. Two new items were added to the original CONSORT for Abstracts statement. The first addresses the reporting of eligibility criteria for centres where the interventions were performed and for care providers. The second addresses the reporting of important changes to the interventions performed versus what was planned.

\section{STROBE for Abstracts}

STROBE for Abstracts is an extension of $\operatorname{STROBE}(8,9)$, the principal reporting guideline for observational studies. In STROBE, the emphasis is on the three main analytical designs found in 
observational epidemiology: cohort, case-control, and cross-sectional studies. Specific items in STROBE for Abstracts cover the potential longitudinal nature of observational studies and ask for a definition of follow-up dates and methods, and key dates at which the outcome events occurred. For studies that rely on matching, such as case-control studies, matching criteria and number of controls per case can also be reported in the abstract. Because observational studies usually do not use randomisation, describing any statistical methods used to adjust for confounding will make the abstract more informative. Of note, STROBE for Abstracts is only available as a draft document.

\section{PRISMA for Abstracts}

PRISMA for Abstracts is an extension of PRISMA $(10,11)$, the main reporting guideline for systematic reviews and meta-analyses, with an emphasis on systematic reviews of interventions. Such studies aim at providing a complete and accurate synthesis of the available evidence regarding a specific clinical question of relevance, most of the time with a quantitative synthesis through meta-analysis. Review authors are encouraged to provide in the abstract a description of databases searched, search dates, and a description of methods for assessing risk of bias in individual trials. Authors are also invited to report the results of the meta-analysis through summary measures of effect size, and to give a summary of the strengths and limitations of their review, including inconsistency and imprecision, indirectness, and risk of bias.

One extension of PRISMA for Abstracts has been published: PRISMA-DTA for Abstracts (25). This checklist preserves the number of items ( $n=12)$, with accommodations specific for systematic reviews of diagnostic accuracy studies. Major changes include: modification of language to diagnostic accuracy lexicon (Item 2: Index test and target condition); removing the item referring to 'effect size'; indicating that risk of bias and applicability should be presented to reflect contemporary guidance for diagnostic accuracy reviews (e.g., QUADAS-2, Quality assessment of diagnostic accuracy studies, the recommended quality assessment tool for diagnostic accuracy studies) (27); and indicating the 
methods used for data synthesis, since diagnostic accuracy systematic reviews require specific random-effects meta-analysis models (e.g., bivariate, hierarchical summary receiver operating characteristic) to account for the correlation between sensitivity and specificity.

\section{STARD for Abstracts}

STARD for Abstracts is an extension of STARD $(12,13)$, which targets diagnostic accuracy studies. These are studies in which the results of one or more (index) tests are compared to the results of the reference standard on the same patients. STARD was initially published in 2003 and was updated in 2015. Specific checklist items invite authors to provide information about the index tests and the reference standard, whether participants formed a consecutive, random, or convenience series, and whether the study was prospective or retrospective. An informative abstract briefly discusses the implications of the results concerning the intended use of the index test (e.g., diagnosis, screening, staging, monitoring, surveillance, prediction, or prognosis). STARD for Abstracts comes with template text for easily writing informative abstracts.

\section{PRIO for Abstracts}

PRIO for Abstracts is an extension of PRIO-harms $(14,15)$, a reporting guideline for overviews of systematic reviews of health care interventions, with an emphasis on harms. Such overviews aim at addressing a broad clinical topic by summarising the evidence from multiple existing systematic reviews, using systematic methods. The checklist includes 20 items in total, but, given usual word limits in journal and conference abstracts, the authors have highlighted 12 items deemed 'highly recommended'. For example, authors are encouraged to report how they assessed the methodological quality of the included reviews (e.g., using AMSTAR, A measurement tool to assess systematic reviews) (28) and the level of quality or certainty of the evidence (e.g., using GRADE, 
Grading of recommendations, assessment, development and evaluation) (29). Authors are also invited to specify in the abstract the method used for evidence synthesis (e.g., narrative, meta-analysis, or network meta-analysis).

\section{DISCUSSION}

Abstracts play a crucial role in how the biomedical information is interpreted and used, but evaluations have shown that their reporting remains suboptimal. We identified five reporting guidelines that can help authors, editors, peer-reviewers, conference organisers, patients, and the general public when reporting and evaluating biomedical abstracts. These five guidelines cover various study designs (i.e., randomised trials, observational studies, diagnostic accuracy studies, systematic reviews, and overviews of systematic reviews). Several extensions of these guidelines have been developed, reflecting more specific study types (e.g., randomised trials of nonpharmacological treatments and systematic reviews of diagnostic accuracy studies). However, several reporting guidelines for main study types (e.g., ARRIVE for animal studies, SQUIRE for quality improvement studies, TRIPOD for prediction models, and COREQ for qualitative research) do not have a corresponding version for abstracts. All guidelines for abstracts are available from the EQUATOR website (www.equator-network.org). They contain 11 to 17 items and are consistent in structure and wording. Only a few items are specific of study type and thus unique to each checklist.

Guidelines for abstracts share a core set of six general items that are likely to be common across all study abstracts. We also identified five generic items that were included in some of the checklists but absent in others. For most of these generic items, there does not seem to be a strong rationale for why they would apply to one type of study and not to others. As the number of reporting guidelines is rapidly expanding, more uniformity across guidelines may be welcomed. The development of reporting guidelines could also be strengthened: protocols were often not available and pilot-testing was rarely reported. 
Developing checklists is certainly not enough to improve the completeness of reporting. The focus should now be on better dissemination, endorsement, and implementation of existing guidance. We should aim at raising authors', peer-reviewers', conference organisers', and editors' awareness of reporting guidelines, and emphasise the importance of using them. A study found that, so far, less than half of journals providing online instructions to authors mentioned reporting guidelines, usually as general statements (30). Thus, as a start, journals and conference organisers are encouraged to officially endorse reporting guidelines for abstracts, by drawing attention to these checklists in their instructions to authors. For example, STARD for Abstracts is now recommended in the abstract submission process for conferences of both the Radiological Society of North America (RSNA) and the European Society of Radiology (ESR).

Empirical evaluations of the actual use and impact of reporting guidelines for abstracts are still scarce. Some authors have investigated adherence $(31,32)$ and its quantitative impact on the informativeness of abstracts, potentially also documenting changes over time (33). Others have evaluated reporting guidelines regarding their usability and acceptability by authors, peer-reviewers, and journal editors. For example, there is evidence that the reporting of abstracts of randomised trials improved after the launch of CONSORT for Abstracts, but a 2012 study found that the guideline improved reporting only when actively implemented at the journal level, for example through emails asking the authors to revise their abstract according to the checklist (33). Furthermore, reporting guidelines are likely to have the most influence and added value when authors are writing rather than when they are submitting their manuscript (34). This suggests that specific training on reporting should not only be offered to editors and peer-reviewers, but also to young scientists (35).

Many journals and conferences have their own requirements regarding the structure and content of abstracts. We also acknowledge that other non-checklist-based guidance for writing abstracts exists and can be of great help to study authors (36). Nevertheless, we encourage journals editors and conference organisers to endorse and explicitly promote the use of these reporting guidelines for 
abstracts, as this will contribute to reducing avoidable waste and to increasing the usability and trustworthiness of reports of health research. 


\section{ADDITIONAL STATEMENTS}

Contributors and sources: JFC and PMB had the idea for the article. The first draft of this article was written by JFC, with feedback and revisions from all the authors, under the supervision of PMB. JFC is the guarantor for this work. JFC's research interests are focused on the methodological aspects of medical test evaluation; he led the development of STARD for Abstracts. PMB leads the STARD Group, and has broader research interests in the evaluation of medical tests. IB is the lead author of the CONSORT extension for nonpharmacological treatments. CG is a member of the STARD Group. SH is the lead author of PRISMA for Abstracts. DAK participated in the development of STARD for Abstracts. MMI is the lead author of the PRISMA extension for diagnostic accuracy reviews. DM is the lead author of PRISMA. EvE is the lead author of STROBE, but was not involved in the development of STROBE for Abstracts. The corresponding author attests that all listed authors meet authorship criteria and that no others meeting the criteria have been omitted.

Funding: There was no specific funding for this study.

Competing interests: All authors have completed the ICMJE uniform disclosure form at www.icmje.org/coi_disclosure.pdf and declare: no support from any organisation for the submitted work; no financial relationships with any organisations that might have an interest in the submitted work in the previous three years; no other relationships or activities that could appear to have influenced the submitted work.

Ethical approval: Not required.

Data sharing: No additional data available.

Transparency: The manuscript's guarantor (JFC) affirms that the manuscript is an honest, accurate, and transparent account of the study being reported; that no important aspects of the study have been omitted; and that any discrepancies from the study as planned have been explained.

Dissemination declaration: Dissemination to study participants and / or patient organisations is not applicable.

Patient and Public Involvement: Patients or the public were not involved in our research. 


\section{BOXES}

\section{Box 1. Methods used to identify reporting guidelines for abstracts}

Search strategy and study selection

Two authors (JFC, DAK) independently searched the website of the EQUATOR network (Enhancing the quality and transparency of health research; www.equator-network.org), an international initiative that promotes transparent and complete reporting in general, and hosts a database of available reporting guidelines. We also conducted unstructured searches on the internet and in MEDLINE. Searches were conducted on August 31, 2019 (last update). To be included in this overview, reporting guidelines had (1) to present a full checklist of items that were deemed essential through any kind of consensus procedure, and (2) to be extensions of existing reporting guidelines for full study reports. Reporting guidelines for full study reports that included some specific items about the abstract, but not a full checklist, were excluded. For included guidelines, we searched for evaluations (adherence, impact, and user satisfaction) in MEDLINE; co-authors' personal files were also screened.

Data extraction and synthesis

For each included reporting guideline, we extracted general characteristics (year published, scope), how the checklist was developed (availability of a protocol, type of consensus process, pilot-testing), type of content (number of items, availability of an explanation and elaboration document and of template text), and whether the guideline had been evaluated (adherence, impact, and user satisfaction). We also extracted individual checklist items. Guideline characteristics were abstracted by one author (JFC) and checked by a second (DAK). Guideline characteristics and items were summarised in tables. We then discussed each guideline in detail and highlighted their specificities. 
Table 1. General characteristics of reporting guidelines for abstracts

\begin{tabular}{|c|c|c|c|c|c|}
\hline & CONSORT for Abstracts & $\begin{array}{c}\text { STROBE for Abstracts } \\
\text { (draft) }\end{array}$ & PRISMA for Abstracts & STARD for Abstracts & PRIO for Abstracts \\
\hline Year published & 2008 & 2011 & 2013 & 2017 & 2019 \\
\hline Scope & $\begin{array}{c}\text { Randomised controlled } \\
\text { trials }\end{array}$ & Observational studies & Systematic reviews & $\begin{array}{c}\text { Diagnostic accuracy } \\
\text { studies }\end{array}$ & $\begin{array}{c}\text { Overviews of systematic } \\
\text { reviews }\end{array}$ \\
\hline \multicolumn{6}{|l|}{ Development } \\
\hline Protocol available & No & No & No & Yes & No \\
\hline Consensus process & $\begin{array}{l}\text { Internet-based survey and } \\
\text { live consensus meeting }\end{array}$ & Unclear & $\begin{array}{l}\text { Internet-based survey and } \\
\text { live consensus meeting }\end{array}$ & Internet-based survey & $\begin{array}{l}\text { Internet-based survey and } \\
\text { discussion until consensus }\end{array}$ \\
\hline Piloting & Not reported & Yes & Not reported & Not reported & Yes \\
\hline \multicolumn{6}{|l|}{ Content } \\
\hline Number of checklist items & 17 & 12 & 12 & 11 & $12^{a}$ \\
\hline Explanation and elaboration ${ }^{b}$ & Yes & No & Yes & Yes $^{c}$ & Yes \\
\hline Template text ${ }^{d}$ & No & No & No & Yes & No \\
\hline \multicolumn{6}{|l|}{ Evaluations $^{\mathrm{e}}$} \\
\hline Adherence (cross-sectional) & Yes & Yes & Yes & Yes & No \\
\hline Impact over time & Yes & No & Yes & No & No \\
\hline User satisfaction & No & No & No & No & No \\
\hline
\end{tabular}

e The full list contains 20 items, among which the authors have identitified 12 'highly recommended' items.

${ }^{b}$ The explanation and elaboration document provides examples of complete reporting and explains the rationale behind each item.

c Provides examples of reporting but refers to STARD 2015 for detailed explanations.

d Template text is standard text that authors can use in their abstract.

e Yes if at least one. 
Table 2. Comparison of items across guidelines: generic items

\begin{tabular}{|c|c|c|c|c|c|}
\hline & CONSORT for Abstracts & STROBE for Abstracts (draft) & PRISMA for Abstracts & STARD for Abstracts & PRIO for Abstracts* \\
\hline \multicolumn{6}{|l|}{ Generic items always present } \\
\hline $\begin{array}{l}\text { Identification of the study with study } \\
\text { design }\end{array}$ & Yes & Yes & Yes & Yes & Yes \\
\hline Study objectives & Yes & Yes & Yes & Yes & Yes \\
\hline Eligibility criteria & Yes & Yes & Yes & Yes & Yes \\
\hline $\begin{array}{l}\text { Number of } \\
\text { participants/studies/reviews }\end{array}$ & Yes & Yes & Yes & Yes & Yes \\
\hline $\begin{array}{l}\text { Results for the primary outcomes and } \\
\text { their precision }\end{array}$ & Yes & Yes & Yes & Yes & Yes \\
\hline General interpretation of the results & Yes & Yes & Yes & Yes & Yes \\
\hline \multicolumn{6}{|l|}{ Generic items sometimes present } \\
\hline Clearly defined primary outcome & Yes & Yes & No & No & No \\
\hline Study setting & Yes & Yes & Not applicable & Yes & Not applicable \\
\hline $\begin{array}{l}\text { Study registration number and } \\
\text { registry }\end{array}$ & Yes & No & Yes & Yes & Yes \\
\hline Source of funding & Yes & No & Yes & No & Yes \\
\hline $\begin{array}{l}\text { Contact details for the corresponding } \\
\text { author }\end{array}$ & Yes & Yes & No & No & No \\
\hline
\end{tabular}

*Here we describe only the 'highly recommended' items. 
Table 3. Comparison of items across guidelines: specific items

\begin{tabular}{|c|c|c|c|c|c|}
\hline & CONSORT for Abstracts & STROBE for Abstracts (draft) & PRISMA for Abstracts & STARD for Abstracts & PRIO for Abstracts* \\
\hline \multicolumn{6}{|l|}{ Specific items } \\
\hline Study design & $\begin{array}{l}\text { Trial design (e.g. parallel, } \\
\text { cluster, non-inferiority) }\end{array}$ & $\begin{array}{c}\text { Study design (e.g. cohort, case- } \\
\text { control, cross-sectional) }\end{array}$ & - & - & - \\
\hline \multirow[t]{3}{*}{ Methods } & $\begin{array}{l}\text { Interventions intended for } \\
\text { each group }\end{array}$ & $\begin{array}{l}\text { Follow-up dates and methods } \\
\text { and key dates at which the } \\
\text { outcome events occurred }\end{array}$ & $\begin{array}{l}\text { Key databases searched and } \\
\text { search dates }\end{array}$ & $\begin{array}{l}\text { Whether this was a prospective } \\
\text { or retrospective study }\end{array}$ & $\begin{array}{c}\text { Key databases searched and } \\
\text { search dates }\end{array}$ \\
\hline & $\begin{array}{c}\text { Randomisation (i.e., how } \\
\text { participants were allocated to } \\
\text { interventions) }\end{array}$ & $\begin{array}{l}\text { For matched studies: matching } \\
\text { criteria, number of controls per } \\
\text { case }\end{array}$ & $\begin{array}{c}\text { Methods of assessing risk of } \\
\text { bias }\end{array}$ & $\begin{array}{c}\text { Whether participants formed a } \\
\text { consecutive, random, or } \\
\text { convenience series }\end{array}$ & $\begin{array}{c}\text { Methods of assessing risk of } \\
\text { bias }\end{array}$ \\
\hline & Blinding (masking) & $\begin{array}{l}\text { Statistical methods used to } \\
\text { control for confounding }\end{array}$ & & $\begin{array}{l}\text { Description of the index test } \\
\text { and reference standard }\end{array}$ & $\begin{array}{c}\text { Method of data synthesis } \\
\text { (narrative, meta-analysis, or } \\
\text { network meta-analysis) }\end{array}$ \\
\hline \multirow[t]{4}{*}{ Results } & Recruitment (trial status) & - & $\begin{array}{l}\text { Direction of the effect (i.e. } \\
\text { which group is favored) and } \\
\text { size of the effect }\end{array}$ & - & $\begin{array}{l}\text { Summary assessment of risk } \\
\text { of bias }\end{array}$ \\
\hline & $\begin{array}{l}\text { Important adverse events or } \\
\text { side effects }\end{array}$ & - & - & - & $\begin{array}{l}\text { Direction of the effect (i.e. } \\
\text { which group is favoured) and } \\
\text { size of the effect }\end{array}$ \\
\hline & - & - & - & - & $\begin{array}{l}\text { Assess the evidence quality } \\
\text { (e.g., GRADE approach) }\end{array}$ \\
\hline & - & - & - & - & $\begin{array}{l}\text { Present harms of the } \\
\text { interventions }\end{array}$ \\
\hline Discussion & - & - & $\begin{array}{l}\text { Strengths and limitations of the } \\
\text { evidence }\end{array}$ & $\begin{array}{l}\text { Implications regarding the } \\
\text { intended use of the index test }\end{array}$ & - \\
\hline
\end{tabular}


1. Scherer RW, Meerpohl JJ, Pfeifer N, Schmucker C, Schwarzer G, von Elm E. Full publication of results initially presented in abstracts. Cochrane Database Syst Rev. 2018;11:MR000005.

2. Hopewell S, Clarke M, Askie L. Reporting of trials presented in conference abstracts needs to be improved. Journal of Clinical Epidemiology. 2006;59(7):681-4.

3. Beller EM, Glasziou PP, Hopewell S, Altman DG. Reporting of effect direction and size in abstracts of systematic reviews. JAMA. 2011;306(18):1981-2.

4. Korevaar DA, Cohen JF, Hooft L, Bossuyt PM. Literature survey of high-impact journals revealed reporting weaknesses in abstracts of diagnostic accuracy studies. Journal of Clinical Epidemiology. 2015;68(6):708-15.

5. Glasziou P, Altman DG, Bossuyt P, Boutron I, Clarke M, Julious S, et al. Reducing waste from incomplete or unusable reports of biomedical research. Lancet. 2014;383(9913):267-76.

6. Schulz KF, Altman DG, Moher D, Group C. CONSORT 2010 statement: updated guidelines for reporting parallel group randomized trials. Annals of Internal Medicine. 2010;152(11):726-32.

7. Hopewell S, Clarke M, Moher D, Wager E, Middleton P, Altman DG, et al. CONSORT for reporting randomised trials in journal and conference abstracts. Lancet. 2008;371(9609):281-3.

8. von Elm E, Altman DG, Egger M, Pocock SJ, Gotzsche PC, Vandenbroucke JP, et al. The Strengthening the Reporting of Observational Studies in Epidemiology (STROBE) statement: guidelines for reporting observational studies. Annals of Internal Medicine. 2007;147(8):573-7.

9. STROBE Group. Draft STROBE checklist for abstracts 2011 [Available from: www.equatornetwork.org/reporting-guidelines/strobe-abstracts].

10. Moher D, Liberati A, Tetzlaff J, Altman DG, Group P. Preferred reporting items for systematic reviews and meta-analyses: the PRISMA statement. Annals of Internal Medicine. 2009;151(4):264-9, W64.

11. Beller EM, Glasziou PP, Altman DG, Hopewell S, Bastian H, Chalmers I, et al. PRISMA for Abstracts: reporting systematic reviews in journal and conference abstracts. PLoS Med. 2013;10(4):e1001419.

12. Cohen JF, Korevaar DA, Gatsonis CA, Glasziou PP, Hooft L, Moher D, et al. STARD for Abstracts: essential items for reporting diagnostic accuracy studies in journal or conference abstracts. BMJ. 2017;358:j3751. 
13. Bossuyt PM, Reitsma JB, Bruns DE, Gatsonis CA, Glasziou PP, Irwig L, et al. STARD 2015: an updated list of essential items for reporting diagnostic accuracy studies. BMJ. 2015;351:h5527.

14. Bougioukas KI, Liakos A, Tsapas A, Ntzani E, Haidich AB. Preferred reporting items for overviews of systematic reviews including harms checklist: a pilot tool to be used for balanced reporting of benefits and harms. Journal of Clinical Epidemiology. 2018;93:9-24.

15. Bougioukas KI, Bouras E, Apostolidou-Kiouti F, Kokkali S, Arvanitidou M, Haidich AB. Reporting guidelines on how to write a complete and transparent abstract for overviews of systematic reviews of health care interventions. Journal of Clinical Epidemiology. 2019;106:70-9.

16. Campbell MK, Piaggio G, Elbourne DR, Altman DG, Group C. Consort 2010 statement: extension to cluster randomised trials. BMJ. 2012;345:e5661.

17. Piaggio G, Elbourne DR, Pocock SJ, Evans SJ, Altman DG, Group C. Reporting of noninferiority and equivalence randomized trials: extension of the CONSORT 2010 statement. JAMA. 2012;308(24):2594-604.

18. Vohra S, Shamseer L, Sampson M, Bukutu C, Schmid CH, Tate R, et al. CONSORT extension for reporting N-of-1 trials (CENT) 2015 Statement. BMJ. 2015;350:h1738.

19. Eldridge SM, Chan CL, Campbell MJ, Bond CM, Hopewell S, Thabane L, et al. CONSORT 2010 statement: extension to randomised pilot and feasibility trials. BMJ. 2016;355:i5239.

20. Boutron I, Altman DG, Moher D, Schulz KF, Ravaud P, Group CN. CONSORT Statement for Randomized Trials of Nonpharmacologic Treatments: A 2017 Update and a CONSORT Extension for Nonpharmacologic Trial Abstracts. Annals of Internal Medicine. 2017;167(1):40-7.

21. Montgomery P, Grant S, Mayo-Wilson E, Macdonald G, Michie S, Hopewell S, et al. Reporting randomised trials of social and psychological interventions: the CONSORT-SPI 2018 Extension. Trials. 2018;19(1):407.

22. Hemming K, Taljaard M, McKenzie JE, Hooper R, Copas A, Thompson JA, et al. Reporting of stepped wedge cluster randomised trials: extension of the CONSORT 2010 statement with explanation and elaboration. BMJ. 2018;363:k1614.

23. Pandis N, Chung B, Scherer RW, Elbourne D, Altman DG. CONSORT 2010 statement: extension checklist for reporting within person randomised trials. BMJ. 2017;357:j2835.

24. Dwan K, Li T, Altman DG, Elbourne D. CONSORT 2010 statement: extension to randomised crossover trials. BMJ. 2019;366:14378. 
25. McInnes MDF, Moher D, Thombs BD, McGrath TA, Bossuyt PM, the P-DTAG, et al. Preferred Reporting Items for a Systematic Review and Meta-analysis of Diagnostic Test Accuracy Studies: The PRISMA-DTA Statement. JAMA. 2018;319(4):388-96.

26. Benchimol El, Smeeth L, Guttmann A, Harron K, Moher D, Petersen I, et al. The REporting of studies Conducted using Observational Routinely-collected health Data (RECORD) statement. PLoS Med. 2015;12(10):e1001885.

27. Whiting PF, Rutjes AW, Westwood ME, Mallett S, Deeks JJ, Reitsma JB, et al. QUADAS-2: a revised tool for the quality assessment of diagnostic accuracy studies. Annals of Internal Medicine. 2011;155(8):529-36.

28. Shea BJ, Reeves BC, Wells G, Thuku M, Hamel C, Moran J, et al. AMSTAR 2: a critical appraisal tool for systematic reviews that include randomised or non-randomised studies of healthcare interventions, or both. BMJ. 2017;358:j4008.

29. Guyatt GH, Oxman AD, Vist GE, Kunz R, Falck-Ytter Y, Alonso-Coello P, et al. GRADE: an emerging consensus on rating quality of evidence and strength of recommendations. BMJ. 2008;336(7650):924-6.

30. Hirst A, Altman DG. Are peer reviewers encouraged to use reporting guidelines? A survey of 116 health research journals. PLoS One. 2012;7(4):e35621.

31. Tsou AY, Treadwell JR. Quality and clarity in systematic review abstracts: an empirical study. Res Synth Methods. 2016;7(4):447-58.

32. Salameh JP, McInnes MDF, Moher D, Thombs BD, McGrath TA, Frank R, et al. Completeness of Reporting of Systematic Reviews of Diagnostic Test Accuracy Based on the PRISMA-DTA Reporting Guideline. Clinical Chemistry. 2019;65(2):291-301.

33. Hopewell S, Ravaud P, Baron G, Boutron I. Effect of editors' implementation of CONSORT guidelines on the reporting of abstracts in high impact medical journals: interrupted time series analysis. BMJ. 2012;344:e4178.

34. Dewey M, Levine D, Bossuyt PM, Kressel HY. Impact and perceived value of journal reporting guidelines among Radiology authors and reviewers. European Radiology. 2019;29(8):3986-95.

35. Chalmers I, Glasziou P. Avoidable waste in the production and reporting of research evidence. Lancet. 2009;374(9683):86-9.

36. Annesley MA. The abstract and the elevator talk: A tale of two summaries (Clinical Chemistry Guide to Scientific Writing). Clinical Chemistry. 2010;56(4):521-4. 